\title{
Pour une recherche sur les routes, voies et réseaux...
}

\author{
S. Robert, N. Verdier
}

Aujourd'hui, il semble que parler de routes et de voies de communication peut relever de deux approches différentes d'abord, et de périodisation fluctuantes ensuite : la première approche serait principalement morphologique, et la seconde principalement fonctionnelle, cela même si une telle coupure est nécessairement fautive. Quant aux périodisations, elles relèvent le plus souvent des périodisations classiques, cela même si on assiste aujourd'hui à une remise en cause de ces découpages.

1. La première approche, ici qualifié de morphologique, décrit les voies de communication et les routes comme un ensemble d'objets dont la matérialité est l'élément le plus fort de la définition. Autrement dit, c'est moins sur la nature de la communication que sur la trace qu'elle “imprime sur le sol”, pour reprendre la métaphore de Paul Vidal de la Blache, que l'on se concentre lorsque l'on évoque ces routes et ces voies. Insister sur la matérialité, sur cette trace qui s'imprime sur le sol, comme la route se dessine sur la carte, c'est le plus souvent accepter l'idée de pratiques régulières qui n'ont dans ce cas de valeur que parce qu'elles s'ajoutent les unes aux autres, qu'elles sédimentent les flux en un cheminement. Comme si la communication devenait voie de communication par l'agrégation de flux quels qu'ils soient.

En dehors de cette sédimentation, l'intérêt pour la matérialité de la route peut relever d'une attention bien spécifique aux pratiques et aux modes de construction de l'objet "route". En France, on est là, les plus souvent du côté d'une histoire de l'institution chargée de cette fabrication, c'est-à-dire le corps des Ponts et Chaussées le plus souvent, ceux qui y sont agrégés, soit par extension vers le passé, soit par absorption d'autres spécialistes. Mais même de ce côté de l'historiographie, deux versants coexistent, le premier valorisant l'invention ou la création, le second l'entretien régulier et répétitif qui fait le gros du travail de l'ingénieur des ponts, autant que du cantonnier. C'est par exemple le cas du bien connu Corps à corps sur la route d'André Guillerme (Guillerme. 1984).

La matérialité, l'impression, la sédimentation, l'agrégation, d'une part, l'entretien répétitif 
d'autre part, autant de façons pour moi d'insister sur l'importance primordiale de l'usage dense et de long terme qui façonne la voie de communication ou la route. Le niveau de cette perception est facilement celui du micro-local. L'un des exemples les plus érudits de ce point de vue est la thèse de Théotiste Gohier sur une route de poste aux chevaux au XVIIIe siècle, pour laquelle l'auteur a reconstruit en très grande partie le tracé de la route à la fin du XVIIIe siècle (Gohier. 1997). C'est en partie ce qui me semble se rapprocher le plus d'une partie des travaux des archéologues qui caractérisent pour partie des voies en fonction de leur usure Dans ce cadre, il est possible de différencier toute une série de tracés sur le sol, du moins affirmé au plus fortement marqué. Le sentier et la piste d'abord qui, pour reprendre une distinction due à Jean-Louis Tissier, "ne relient pas des points correspondants à des centres de peuplement permanents" (Tissier. 2003). Ceux-là ne permettent pas une sédimentation par la médiocre quantité de flux qu'ils subissent. Le plus souvent, ils nécessitent des guides, capables de lire des traces invisibles au voyageur. Ils se composent de point de passage, disséminés de loin en loin sur un terrain peu différencié, du moins sous l'angle des indices viaires. Ici, c'est à la belle publication du colloque sur les guides d'il y a quelques années qu'il faut renvoyer (Chabaud et al. 2000). La bibliographie à prendre en compte relève de la question des points de passage, très rarement le tracé est prix en compte. Au-dessus des sentiers et des pistes apparaissent les chemins, qui lient des centres de peuplement permanent. Ici les guides perdent leur fonction. Les échanges entre lieux habités laissent des traces tellement évidentes sur le sol que n'importe qui peut distinguer le chemin dans le paysage. Reste la route qui se différencie par sa stature et son statut. Par stature, entendons sa largeur qui le plus souvent, en dehors du cas toujours cité des drailles (chemin sur lequel transitent de vastes troupeaux), permet de séparer nettement la route des chemins. Par statut ce qu'il faut comprendre c'est l'officialisation précoce de la route par l'autorité politique : la route est une voie d'État.

On pourrait essayer de préciser que le statut de la route n'est pas si simple, certaines routes n'étant pas purement d'État, mais pour finir, il me semble que nous pourrions nous accorder sur l'idée que la route est un chemin marqué dans le paysage auquel on accorde une fonction qui rejaillit sur sa forme (largeur, matériau, bornes, lieux d'étapes, etc.). Il en est ainsi des voies romaines et des routes de Poste, même si ces dernières se distinguent des routes royales, voire des routes de marche des armées. Les routes de Poste sont des routes dénommées ainsi par une institution privée (qui soit détient un monopole). Les routes de marche des armées ne prennent leur forme complète que lorsqu'elles sont utilisées, tout comme les routes royales qui le deviennent en fonction des besoins de transport des rois de France. La route serait alors 
un objet intermittent, changeant de statut lorsqu'une mission lui est attribuée. Le principe d'autorité semble un élément majeur de la définition routière. De ce point de vue la bibliographie est pour le moins limitée, en dehors de quelques pages dans l'historiographie relative aux Ponts et Chaussées.

2. La définition concurrente au principe d'autorité hiérarchise les usages. On la trouve, par exemple chez le géographe Capot-Rey dans les années 1940 lorsque celui-ci fait du chemin le lieu de déplacement à pieds, ou à cheval, mais fait de la route celui des voitures, quelles qu'elles soient (Capot-Rey, 1946). Selon Capot-Rey, la roue fait la route et l'automobile fait l'autoroute. Il y aurait donc une hiérarchisation des voies de communication qui suivrait sans le dire un progrès technique, allant du sentier pour le piéton à l'autoroute de l'automobiliste. À ce titre, il ne peut y avoir de route de la soie, ou de route des épices, mais il y aurait des chemins muletiers. De même, le chemin de fer, n'est qu'une voie de communication de plus pas réellement séparée des autres, cela alors que, la plupart du temps, les spécialistes des routes laissent de côté les voies ferrées.

Cette approche par la matérialité, que nous avons qualifié de morphologique a été critiquée très tôt. Lucien Febvre par exemple, même s'il était sensible au discours des géographes n'en préfère pas moins une approche fonctionnelle de la route (Febvre. 1922). Rejetant toute forme de déterminisme au profit du possibilisme, il préfère poser la question des raisons pour lesquelles l'homme se déplace plutôt que de s'appesantir sur l'agrégation des flux sur un temps plus ou moins longs. Ce faisant, ce que Febvre opère est clairement une séparation entre la route et le sol. Ce qui importe n'est pas de savoir s'il y a beaucoup de passages, mais de connaitre les causes de ces passages. Pour Febvre, il n'y a de route que parce qu'il y a une volonté humaine. Exit la matérialité : la route de la soie, même lorsqu'elle vit sur une contrée non aménagée, entendons sans étapes constituées à son usage propre, reste une route puisqu'elle a une fonction. Dès lors l'historicité se met en place puisque les fonctions changent dans le temps, les routes changent également. On retrouve ici la route en tant que construction liée à l'État puisque pour Febvre, la route est "fédératrice et génératrice d'État". Selon Febvre, à l'origine des routes, on trouve "une activité réfléchie, une intelligence créatrice, une volonté éprouvée aux prises avec les puissances obscures du milieu". Ici, la matérialité des routes n'a d'importance qu'en ce qu'elle permet de mesurer la puissance de la fonction confrontée à ces "puissances obscures du milieu". La route est le témoignage de l'action de l'État. 
3. Au-delà de ces deux approches, reste la question encore ouverte de la périodisation des études routières. Traditionnellement la recherche sur le réseau routier s'est trouvée sectorisée dans différentes disciplines en fonction de sources et de périodes chronologiques définies. Devant l'importance des vestiges antiques conservés, les archéologues se sont focalisés dès le XVIIe siècle sur l'étude des voies de cette période. En fonction des textes conservés, les historiens se sont penchés surtout sur les usages liés aux grandes routes médiévales. Quant au réseau moderne et contemporain, bien documenté, il a été étudié par des historiens et des géographes à partir d'archives ou de textes publiés, bien souvent par l'intermédiaire d'analyses cartographiques ou statistiques.

Ces dernières années, l'approche archéogéographique a remis quelque peu en cause cette répartition, en étudiant les routes anciennes à partir des sources planimétriques modernes et contemporaines. Cadastres, cartes topographiques ou photographies aériennes montrent en effet de manière spectaculaire la transmission d'une voie antique par exemple, dans la succession d'une route actuelle, d'un chemin, d'une haie ou d'une limite communale, dépassant ainsi l'approche morphofonctionnelle classique. Des concepts tels que réseau, résilience, auto-organisation etc. se sont vus transférer depuis la géographie vers l'archéologie et l'utilisation des systèmes d'information géographiques et de la modélisation spatiale a encore rapproché les deux disciplines autour d'outils communs.

Depuis quelques années, l'étude des réseaux routiers a permis de renouveler le concept de transmission des formes en archéogéographie. Des travaux d'E. Vion à la fin des années 1980 (Vion. 1989) aux thèses de C. Marchand (Marchand. 2000) et S. Robert (Robert. 2004) au début des années 2000, l'étude des réseaux routiers est apparue comme un élément heuristique pour l'étude des phénomènes de transmission des formes dans la longue durée. C'est un exemple particulièrement parlant de la résilience des formes dans le temps, c'est-àdire leur capacité à maintenir leur identité structurelle et fonctionnelle même après avoir intégré d'importantes perturbations. Plusieurs des itinéraires étudiés laissent apparaitre une longévité remarquable depuis l'antiquité voire la protohistoire.

Enfin, depuis les années 1990, les découvertes de terrain réalisées dans le contexte de l'archéologie préventive ont été très nombreuses et amènent de nouveaux éclairages et questionnements. Elles permettent de renouveler la connaissance sur les routes anciennes, encore fortement héritière des recherches effectuées au XIXe siècle et dans les années 1970. Aujourd'hui, un grand nombre de propositions de tracés reste principalement issu de ces deux premières phases de recherche sur les voies. 
4. La volonté d'aborder différemment les réseaux routiers, de bénéficier des apports récents de l'archéogéographie et de l'archéologie préventive et de favoriser les échanges entre disciplines s'est traduite en 2005, par la constitution d'un groupe de recherche autour de la dynamique des réseaux routiers du Bassin Parisien associant historiens archéologues et géographes dans une approche diachronique. Il s'est formalisé en 2007 sous la forme du Programme Collectif de Recherche Dynarif (Dynamique et résilience des réseaux parcellaires et viaires en Région Ile-de-France) associant des membres du CNRS (UMR 7041 Arscan et UMR 8504 : Géographie-cités), des Universités Paris I et Paris X, de la DRAC-SRA Ile-deFrance, , du IGN - COGIT, de l'INRAP, des Conseils Généraux du Val-d'Oise, des Yvelines, de Seine-et-Marne, de Seine-Saint-Denis, du Val-de-Marne et des Hauts-de-Seine et de la Ville de Paris. Ce projet vise la reconstitution des réseaux routiers et une analyse diachronique des réseaux depuis la Protohistoire jusqu'à la période moderne en Ile-de-France, à travers une base de donnée construite sur un système d'information géographique (axe 1 du PCR). Cette grande marge chronologique doit permettre d'étudier la dynamique de formation et d'évolution du réseau, plus particulièrement les phénomènes de transmission et de résilience dans le temps. Un apport essentiel est l'apport des données d'archéologie préventive. L'attention est attirée sur les voies de grand parcours non monumentales qui ont été peu étudiées jusqu'à maintenant et contribuent pourtant à dégager les réseaux protohistoriques et médiévaux. À l'échelle micro-régionale, sur des zones tests, les interactions entre voies, établissements, parcellaires et environnement sont observées pour montrer le rôle morphogénétique des axes de circulation dans la construction des réseaux spatiaux et des paysages (axe 3). Cet axe du PCR est développé en collaboration avec le programme ANR «Alpage », spatialisation des sources historiques sur la ville de Paris (Université Paris I, LAMOP, Université de La Rochelle), dirigé par H. Noizet et le Projet d'Activités Scientifiques «Evolution des terroirs au travers des opérations de grande ampleur: dynamique d'évolution du plateau de Sénart à travers les âges » (INRAP), dirigé par J. Legriel, L. Boulenger et S. Robert.

Enfin, une réflexion est menée sur le vocabulaire à appliquer à la recherche sur les réseaux routiers (axe 2). Il s'agit de tenter, si ce n'est de fixer le vocabulaire, ce qui scléroserait probablement la recherche, au moins de parvenir à une meilleure compréhension des différents types de vocabulaires employés. On l'a vu plus haut, les mots même de route, voire de celui de chemin ou de réseau méritent de nombreux approfondissements. Quant à ceux de route arpentée, de voies monumentales d'une part, de circulation, de hiérarchisation des voies d'autre part, et les listes sont loin d'être exhaustives, la pratique interdisciplinaire en a 
rapidement montré les limites dans la mise en miroir de conception différentes voire opposées. Enfin, un dernier axe insiste sur la modélisation des tracés théoriques à confronter à la base de données réalisées (axe 4). L'objectif est clairement de tenter de maîtriser au mieux les méthodes à importer pour en tirer le plus grand profit possible.

Ce numéro spécial des Nouvelles de l'archéologie constitue un état de la réflexion après deux ans d'échanges au sein du groupe de recherche et l'organisation de trois journées d'étude qui ont porté sur :

- 'Les approches diachroniques sur l'analyse des réseaux routiers en Île-de-France et Normandie", journée organisée par S. Robert le 15 juin 2006 à la Maison de l'Archéologie et de l'Ethnologie de Nanterre, dans le cadre du Programme « Archéologie du Bassin Parisien », dirigé par P. Brun et P. Soulier (UMR 7041).

- "La croisée des chemins. Routes et voies de communication au crible de l'interdisciplinarité", journée organisée le 13 novembre 2006 à l'EHESS par N. Verdier, dans le cadre du Groupe de Géographie et d'Histoire Urbaine de l'EHESS, de l'UMR 8504 Géographie-cités (CNRS) et du Master en Sciences Sociales de l'EHESS, mention Territoire Espace Société.

- «Réflexion sur la modélisation des réseaux routiers en archéologie » journée organisée par S. Robert et N. Verdier le 13 février 2008, à la Maison de l'Archéologie et de l'Ethnologie (Nanterre), dans le cadre du PCR Dynarif et du Master 2 « Archéologie et Environnement », option Archéogéographie de l’Université de Paris I.

5. Au cours de ces échanges, archéologues, historiens et géographes se sont retrouvés autour de plusieurs idées fortes qui constituent le lien entre les différentes contributions présentées ici.

Lorsque l'on dépasse la vision de la route comme monument (S. Robert) et que l'on s'attache au réseau dans son ensemble, se dégage son caractère fortement anastomosé (C. Marchand, S. Robert, N. Verdier). Les nœuds du réseau semblent plus pérennes que les tronçons qui ne cessent de changer dans le temps. Stéphane Blond montre de ce point de vue que l'Atlas Trudaine porte témoignage d'un moment fort de labilité du réseau : lorsque de nouvelles voies, plus rectilignes en remplacent d'autres. Pour le cas de la poste aux chevaux par exemple, N. Verdier montre que ce ne sont que quelques relais, parfois urbains, qui sont fixes dans le temps long et qui font l'objet de l'attention des pouvoirs publics plus que les tronçons extrêmement labiles. La théorie des graphes, conceptualisant les relations complexes entre 
lignes et nœuds, fournit des outils pour étudier le réseau (cf. A. Chevalier et J. F. Gleyze). De ce point de vue les textes de Jean-François Gleyze et d'Arnaud Chevalier sont à voir comme les deux aspects d'un même raisonnement. Le texte de Jean-François Gleyze, offre ici dans une présentation limpide les apports possibles d'une analyse exigeante des réseaux, tels qu'ils peuvent être employés lors de synthèses des données archéologiques. Celui d'Arnaud Chevalier en montre une application fine qui lie intimement enquête de terrain et analyse spatiale. Pour autant, on ne peut se contenter d'observer de simples liaisons théoriques entre des points. L'analyse de la structure matérielle existante du réseau est déterminante pour sa résilience dans le temps. Son caractère anastomosé intervient notamment dans la vulnérabilité du réseau (J.-F. Gleyze) et est garante de sa résilience dans le temps (S. Robert, C. Marchand). La forme du réseau, dont une grande partie est héritée, lui confère des propriétés et des potentialités notamment en termes d'accessibilité et de centralité des lieux (J. F. Gleyze et N. Verdier).

Le rôle structurant des réseaux routiers dans l'organisation du peuplement et du paysage est largement démontré. En utilisant la notion de percolation, A. Chevalier montre que la route agit notamment dans la distribution des tumuli protohistoriques dans la plaine de Caen. Les chemins de grand parcours jouent également un rôle déterminant dans l'organisation des trames parcellaires et cela dès la protohistoire (C. Marchand et S. Robert). La relation est donc complexe entre les différentes composantes du réseau. Les nœuds contribuent au maintien des lignes tout en étant fortement influencés par elles.

Etudier la dynamique du réseau dans le temps ne peut faire l'économie d'une réflexion sur les échelles spatiales. En effet, les rythmes peuvent être très différents à l'échelle des itinéraires régionaux et à celle des tracés locaux. La mobilité ou la pérennité qui s'observe à une échelle ne peut se généraliser à l'autre (S. Robert, N. Verdier). Pour autant, les différentes échelles n'agissent pas indépendamment les unes des autres. Un jeu d'interactions complexes se tisse entre le local et le global, l'un étant souvent la condition de pérennisation de l'autre : le tracé de grand parcours attire et contribue à la fixation de l'habitat local et en même temps, ce dernier entretient le réseau de grand parcours et participe à sa pérennisation dans le temps $(\mathrm{C}$. Marchand et N. Verdier).

La question des échelles oblige à s'interroger sur les limites des sources utilisées. En archéologie, on déduit trop souvent des interprétations fonctionnelles et chronologiques sur un itinéraire global à partir d'observations très localisées. Pourtant les critères matériels seuls ne peuvent permettre de déterminer le statut d'une voie et l'observation en un point donné de critères chronologiques ne vaut que pour le tronçon et non pour l'ensemble de l'itinéraire. 
Seul des cas de transformations généralisées du système routier, comme celui dépeint par Stéphane Blond, nous offre un état des lieux, à un moment donné. Encore, les planches de l'Atlas Trudaine sont-elles à prendre avec précaution, et toujours à comparer avec des données issues du terrain. Les observations de terrain peuvent être croisées alors avec les données archéogéographiques pour dépasser la fenêtre de la fouille et être hiérarchisées (S. Robert). De même, il faut distinguer les informations fonctionnelles sur le réseau et la structure matérielle (J. F. Gleyze). Le croisement de la modélisation spatiale des relations entre les sites archéologiques (à travers des modèles de diffusion par exemple) et la reconstitution archéogeographique ouvre un nouveau champ de possibilités pour l'étude des réseaux anciens.

Le réseau structurel existant apparaît déterminant dans sa dynamique fonctionnelle. Son accroissement est fortement dépendant de l'effort à fournir pour le maintien de l'existant (N. Verdier). L'héritage est important et l'interaction entre différents niveaux d'acteurs ne permet plus d'écrire l'évolution du réseau routier de grand parcours uniquement en fonction de celle des pouvoirs publics susceptibles de l'aménager et le maintenir dans le temps. Le rôle des périodes protohistoriques et médiévales, traditionnellement considérées comme des phases pauvres ou de rupture dans la constitution des réseaux routiers, faute de l'existence d'un pouvoir centralisé fort, doit être réévalué (S. Robert). Les nombreuses découvertes en archéologie de terrain et en archéogéographie ont fourni ces dernières années de nouveaux objets: chemins protohistoriques et médiévaux de grand parcours, voies antiques non arpentées.... Reste aujourd'hui à les inventorier et à les étudier plus systématiquement.

Une histoire par rupture n'est donc plus possible aujourd'hui mais pour autant, il ne s'agit pas d'une histoire immobile. Les articles regroupés ici soulignent la labilité de certaines des composantes du réseau et montrent que cette mobilité incessante permettant créations, réinvestissements, transformations, échappée en cas de rupture garantit la stabilité de l'ensemble (S. Blond, J. F. Gleyze, C. Marchand, S. Robert et N. Verdier). Cette vision du réseau qui permet d'associer de manière dynamique différentes échelles spatiales et temporelles d'étude permet aujourd'hui ce rapprochement entre disciplines. 
Bibliographie du dossier Des Nouvelles de l'Archéologie «Du sentier à la route, une archéologie des réseaux viaires » coordonné par Sandrine Robert et Nicolas Verdier.

Agache, R. 1978. La Somme pré-romaine et romaine. Amiens, Société des Antiquaires de Picardie, $515 \mathrm{p}$.

Allen, W. B., Liu, D., Singer, S. 1993. "Accessibility measures of US metropolitan areas », Transportation Research, 27B (6) : 439-449.

Les Annales, 1989., “Tentons l'expérience ”, Annales E.S.C. nº. 1317-1323.

Antolne A. 2000. Le paysage de l'historien, Archéologie des bocages de l'Ouest de la France à l'époque moderne, Rennes, Presses universitaires de Rennes. 344 p.

Antorne, A., Vennel J.-P. 1994. « La voie royale et le paysage », Société archéologique et d'histoire de la Mayenne, fascicule 17. 35-57.

Arbellot G. 1973. « La grande mutation des routes de France au milieu du XVIII ${ }^{\mathrm{e}}$ siècle », Annales Économies Sociétés Civilisations, $\mathrm{n}^{\circ} 3$ : 765-791.

Arbellot, G. 1980. « Le réseau des routes de poste, objet des premières cartes thématiques de la France moderne ", 104e congrès des sociétés savantes, Bordeaux 1979, Histoire moderne, Paris, Bibliothèque Nationale, 1 : 97-115.

Auray, J.-P., Bailly, A., Derycke, P.-H., Huriot, J.-M. 1994. Encyclopédie d'économie spatiale Concepts - Comportements - Organisations. Economica, Paris, 427 p.

Bairoch, P., Batou, J. et Chèvre, P. 1988. La population des villes européennes de 800 à 1850, Genève, Droz.

Balard, M. dir., Laurioux, B. Le Jan, R., Le Mené, M. 2003. Dictionnaire de la France médiévale. Paris, Hachette, 287 p.

BAUtier, R. H. 1987. « La route française et son évolution au cours du Moyen Age », Académie royale de Belgique, Bulletin de la classe des lettres et sciences morales et politiques, 1987, t. 73 : 70-104.

Benort, F. 1998. Modes d'occupation de l'espace protohistorique dans le Bassin moyen de l'Aisne, Mémoire de Maîtrise d'Archéologie, Université Panthéon-Sorbonne Paris I, 252 p.

Berge, C. 1973. Graphes. Éditions Gauthier-Villars, Paris, 400 p.

Berger, J. F., Bertoncello, F. , Braemer, F., Datvian, G., Gazenbeck, M. (dir.) 2005. Temps et Espaces de l'Homme en société, analyses et modèles spatiaux en archéologie. XXVe rencontres internationales d'archéologie et d'histoire d'Antibes, Antibes, Editions APDCA, 543 p. 
Bertrand, A.1864. «Voies romaines en Gaule, voies des itinéraires. Résumé du travail de la Commission de la topographie des Gaules », Extrait de la Revue archéologique. Paris, Librairie académique, $61 \mathrm{p}$.

Bradley, R. 1991. «The pattern of change in British prehistory », in : T. Earle (ed.), Chiefdoms: power, economy and ideology. Cambridge : 44-70.

Bradley, R. et Hart, C. 1983. "Prehistoric Settlement in the Peak District during the Third and Second Millennia bc: A Preliminary Analysis in the Light of Recent Fieldwork », Proceedings of the Prehistoric Society, 49 : 177-193.

BRÉCHON, F. 2000. Réseau routier et organisation de l'espace en Vivarais et sur ses marges au MoyenAge. Thèse d'histoire. Lyon, Université de Lumière Lyon 2, 1368 p.

Bretagnolle, A. et Verdier, N. 2007. « L'extension du réseau des routes de poste en France de 1708 à 1833 », in Le Roux, M. (dir.), Postes d'Europe XVIIIe-XXIe siècles. Jalons d'une histoire comparée, Paris, Comité pour l'Histoire de la Poste, 2007, 155-171.

BRUAND, O. 2002. Voyageurs et marchandises aux temps carolingiens: les réseaux de communication entre Loire et Meuse aux VIIIe et IXe siècles. Bruxelles, De Boeck Université, 357 p.

Bruinsma, F., Rietveld, P. 1993. «Urban agglomerations in European infrastructure networks », Urban Studies, 30 : 919-934

Brun, P., Guichard, Y. et Le Goff, I. 2005. « Les tombes à incinération de l'âge du Bronze et du Ier âge du Fer dans le bassin de l'Aisne : Observations préliminaires. », in : Les pratiques funéraires à l'âge du Bronze en France, Actes de la table ronde de Sens-en-Bourgogne, 10-12 juin 1998. Société Archéologique de Sens, Comité des travaux historiques et scientifiques : 477-492.

Burgess, C. 1980. The Age of Stonehenge. Londres, J.M. Dent \& Sons Ltd, 402p.

CAPOT-Rey, R. 1946. Géographie de la circulation sur les continents, Paris, Gallimard, 296 p.

Castex, J, Céleste P. et Panerai Ph. 1979. Lecture d'une ville : Versailles. Paris, Editions du Moniteur, col. Architecture "Etudes", 235 p.

Cattan, N., Grasland, C. 1997. Les différentiels d'accessibilité des villes moyennes en France. Rapport de recherche pour le METL, CNRS, Laboratoire ParisGeo, 115p.

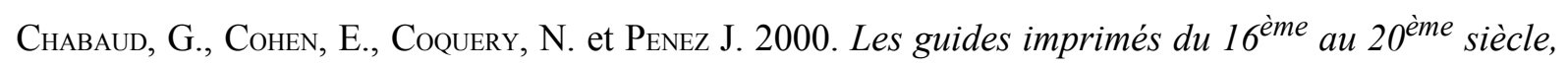
villes, paysages voyages, Paris, Belin, $704 \mathrm{p}$.

ChApelon, L. 1997. Offre de transport et aménagement du territoire - Evaluation spatio-temporelle des projets de modification de l'offre par modélisation multi-échelles des systèmes de transport. Thèse de doctorat en Aménagement, Université François Rabelais de Tours, 558 p. 
Снарецот, J. 1993. "L'habitat rural : organisation et nature », in L'Ile-de-France, de Clovis à Hugues Capet, du Ve au Xe siècle, catalogue de l'exposition, Guiry-en-Vexin, Musée archéologique, 178-199. Снаротат, G. 1981. «La voie protohistorique sud de la croisée de Vienne. Essai de reconstitution de son tracé jusqu'à Marseille ». Revue Archéologique de l’Est, 1981, Tome XXXII, n¹25-126 : 83-91

Chartrain, A. 2007. «Parcellaires fossiles et faits funéraires dans l'espace et le temps sur le territoire rural de Montpellier » Medieval Europe Paris 2007, http://www.archeogeographie.org

Chevalier, A. 2005. Les enclos funéraires protohistoriques de Basse-Normandie : Essai d'analyse spatiale, Mémoire de Maîtrise d'Archéologie, Université Panthéon-Sorbonne, Paris I. 175 p.

Chevalier, A. 2006. Recherche d'une structuration de l'espace protohistorique en Basse-Normandie : exercice d'analyse spatiale, Mémoire de Master II Recherche, Université Panthéon-Sorbonne, Paris I. $125 \mathrm{p}$.

Chevallier, R. 1972. Les voies romaines. Paris, Armand Colin, 313 p.

Chevallier, R. 2000. Lecture du temps dans l'espace. Topologie archéologique et historique Paris, Picard, 229 p.

Chevallier, R. dir. 1983. Les voies anciennes en Gaule et dans le monde romain occidental, Colloque tenu à Paris, ENS, 5-6 juin 1982 ». Caesarodunum nXVIII, Tours, Université de Tours, 487 p.

Chouquer, G. (dir.) 1996. Les Formes du paysage, Tome I : études sur les parcellaires : pré-actes du colloque d'Orléans (mars 1996). Paris, éditions Errance, 222 p.

Chouquer, G. (dir.) 1996. Les Formes du paysage, Tome.2: Archéologie des parcellaires, Paris, Editions Errance, 263 p.

Chouquer, G. (dir.) 1997. Les Formes du paysage, Tome 3 : L'analyse des systèmes spatiaux, Paris, Editions Errance, 198 p.

Chouquer, G. 1996. «Parcellaires et longue durée; Points de repères historiques et problèmes d'interprétation », in: Chouquer G. dir. Les Formes du paysage, Tome 2 : Archéologie des parcellaires. Actes du colloque d'Orléans (mars 1996). Paris, Editions Errance, 1996 : 201-223.

Chouquer, G. 2000. L'étude des paysages. Essais sur leurs formes et leur histoire. Editions Errance.

Paris. 208 p.

Chouquer, G. 2006. «L'émergence de la planimétrie agraire à l'Age du Fer », in : Chouquer G. dir. Etudes Rurales $\mathrm{n}^{\circ} 175-176: 29-52$.

Chouquer, G. et al. 1996. « La morphologie agraire et les paysages de la plaine des Tilles et de l'Ouche (Côte-d'Or) ». Chouquer, G. (dir.) Les Formes du paysage, Tome I : études sur les parcellaires : préactes du colloque d'Orléans (mars 1996). Paris, éditions Errance,: 32-49. 
Coquery, N. L'Hôtel Aristocratique. Le marché du luxe à Paris au XVIIIe siècle, Paris, Publications de la Sorbonne. 444 p.

Dainville F. (de) 1964. Le langage des géographes, termes signes, couleurs des cartes anciennes, 1500-1800, Paris, Picard. XX-384 p.

Daremberg, Ch. et SAglio, E. 1969. Dictionnaire des antiquités grecques et romaines : d'après les textes et les monuments. Paris, Hachette, pp. écrit entre 1877 et 1906, 10 vol. $1^{\text {ère }}$ édition 1934.

Daveau, I. et Yvinec, J. H. 2002. «L'occupation gallo-romaine du site de Fontenay-en-Parisis "La Lampe" (Val-d'Oise) : établissement agricole spécialisé ou lieu de culte ?» in : Revue Archéologique du Centre, Tome 41, Tours, $2002:$ 129-172.

Decoupigny, C. 2003. «Couloirs de trafics et émissions de polluants », in: Mathis, Ph. (dir.) 2003. Graphes et réseaux, modélisation multiniveau. Paris, Lavoisier: 187-211.

Demangeon, P. 1995. La Poste en Basse Normandie, Vire, Charles Corlet éditions. 439 p.

Denford, G.T. 1975. "Economy and location of Bronze Age "arable" settlements on Dartmoor », Bulletin of the Institute of Archaeology, 12 : 175-196.

DeRVILLE, A. 1978. «La première révolution des transports continentaux, (c.1000 - c. 1300.) », in : Annales de Bretagne et des Pays de l'Ouest 85 (1978) : 181-205

Desbordes, J. M. et Barrière, B. 1983. "Anciens itinéraires entre Limousin et Périgord », in: Chevallier, R. dir. 1983. "Les voies anciennes en Gaule et dans le monde romain occidental, Colloque tenu à Paris, ENS, 5-6 juin 1982 ». Caesarodunum $n^{\circ} X V I I I$, Tours, Université de Tours, : 189-199.

DesLoges, J. à paraître. Histoire du peuplement de la Basse-Normandie, L'apport de la prospection aérienne 1988 - 2004. Direction Régionale des Affaires Culturelles de Basse-Normandie, Service Régional de l'Archéologie.

Devroey, J. P. 1984. «Un monastère dans l'économie d'échanges : les services de transport à l'abbaye Saint-Germain-des-Prés au IXe siècle », in : Annales Economie Sociétés Civilisation, 39, 1984 : 570 589

Dion, R. 1961. Histoire des levées de la Loire, Paris, 312 p.

Dindjian, F. 1991. Méthodes pour l'archéologie. Paris, Armand Colin, 401 p.

Dunin-Wasowicz, T. 1982. «Les routes médiévales en Europe centrale : bilan et perspectives », in :

Durand-Dastès, F. et al. 1998. Archaeomedes. Des oppida aux métropoles. Archéologues et géographes en vallée du Rhône. Paris, Anthropos, Economica, 280 p.

EJSTRud, B. 2005. «Cost surface analysis and ancient roads : a comparison », in : Berger, J. F., 
Bertoncello, F. , Braemer, F., Datvian, G., Gazenbeck, M. (dir.) 2005. Temps et Espaces de l'Homme en société, analyses et modèles spatiaux en archéologie. XXVe rencontres internationales d'archéologie et d'histoire d'Antibes, Antibes, Editions APDCA : 135-139.

FAVIER, J. 1993. Dictionnaire de la France médiévale. Paris, Fayard, 982 p.

Favory, F. , Fiches, J.L., Raynaud, C. et Clatot, M. « La dynamique de l'habitat gallo-romain dans la basse vallée du Rhône », in DuRAnd-DAstès, F.et al. 1998. Archaeomedes. Des oppida aux métropoles. Archéologues et géographes en vallée du Rhône. Paris, Anthropos, Economica : 73-115.

Favory, F., Parodi, A., Poupet, P. et Raynaud, C. 1994. «Lunel-Viel et son territoire », in : Favory F. et Fiches J. L. (dir.). Les campagnes de la France méditerranéenne dans l'Antiquité et le haut MoyenAge, DAF n², Paris, Editions de la maison des sciences de l’Homme, 1994 : 163- 244

FeBVRE, L. 1922. La terre et l'évolution humaine, introduction géographique à l'histoire, Paris, Renaissance du livre, $470 \mathrm{p}$.

Fourquin, G. 1964. Les campagnes de la région parisienne à la fin du Moyen-Âge, Paris, PUF, 590 p.

Freeman, L. 1979. «Centrality in social networks - Conceptual clarification ». Social Networks, n¹ : 215-239

Garmy, P., Kaddouri, L., Rozenblat C. et Schneider, L. 2005 « Logiques spatiales et « systèmes de villes » en Lodévois de l'Antiquité à la période moderne », in Berger, et al. (dir.). Temps et Espaces de l'Homme en société, analyses et modèles spatiaux en archéologie. XXVe rencontres internationales d'archéologie et d'histoire d'Antibes, Antibes, Editions APDCA : 225-236.

Gleyze, J.-F. 2005. La vulnérabilité structurelle des réseaux de transport. Thèse de doctorat de Géographie - Université de Paris VII Denis Diderot, 848 p.

Gleyze, J.-F. 2007a. « Effets spatiaux et effets réseaux dans l'évaluation d'indicateurs sur les nœuds d'un réseau d'infrastructures ». CyberGeo, n³70, 24 p. http://www.cybergeo.eu/

GleYZE, J.-F. 2007b. « Reducing functional network vulnerability - Structural indicators to anticipate the structural reorganization of a damaged transportation network ", in : Actes de la 15ème édition de la Conférence Européenne de Géographie Théorique et Quantitative - ECTQG, Montreux, Suisse, 4 p.

GoHIER, Th. 1997. La poste aux chevaux dans la région malouine (1738-1870), Thèse de doctorat de Sciences Sociales de l'universtié de Haute-Bretagne Rennes II, sous la direction de Claude Nières, 1997, 2 vol.

Gould, S.J. 2005. Le renard et le hérisson, Comment combler le fossé entre la science et les humanités. Trad. N. Witkowski, Paris, Seuil, 364 p. 
Grenier, A. 1985. Manuel d'archéologie gallo-romaine. Tome 2, L'archéologie du sol. Navigation-

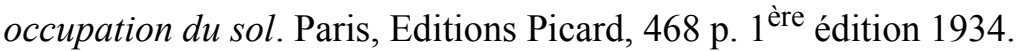

Groupe Dupont, 2004, Géopoint 2004, La forme en géographie, Avignon, Groupe Dupont, 505 p.

Guilaine, J. 1991. «Vers une préhistoire agraire » in: J. Guilaine (dir.), Pour une archéologie agraire, Paris, Armand Colin : 31-80.

Guilaine, J. 2000. « Changeons d'échelles : pour la très longue durée, pour de larges espaces », Etudes rurales, 153-154:9-21.

Guillaumet, J. P. 1996. L'artisanat chez les gaulois. Paris, Errance, 127 p.

Guillerme, A., 1984, Corps à corps sur la route. Les routes, les chemins et l'organisation des services $d u$ XIXe siècle, Paris, Presses de l'ENPC, 172 p.

Herce Vallejo, M. 1983. "La utilización de indicadores topológicos en el analisis de redes de communicaciones - Ensayo sobre la red de carreteras de Cataluña ». Documents d'Anàlisi Geogràfica, $n^{\circ} 3: 3-46$

Herrmann, P. 2007. Itinéraires des voies romaines. Paris, Editions Errance, 275 p.

Hodson, F.R. 1971. «Cluster analysis Numerical typology and prehistoric archaeology », in : F.R. Hodson, D.G. Kendall et Tautu P. (éds), Mathematics in the Archaeological and Historical Sciences. Proceedings of the Anglo-Romanian Conference, Mamaia 1970, organized by The Royal Society of London, and The Academy of the Socialist Republic of Romania, Edinburgh, Edinburgh University Press : $30-45$.

HuRE, A. 1978. Le Sénonais gallo-romain, Bruxelles, Editions Culture et civilisation, 422 p.

Huriot, J.-M., Perreur, J. 1994. « La centralité / L'accessibilité », in : (Auray J.-P. et al. 1994) : 47-58.

InGRAM, D.R. 1971. "The concept of accessibility: a search for an operational form ». Regional Studies, $5: 101-107$

JEANNET, N. (à paraître). «Contributions pratiques d'une géostatistique raisonnée en environnement : méthodes et application à la cartographie régionale de la pollution par le dioxyde d'azote $\left(\mathrm{NO}^{2}\right)$ en Alsace », in : Aubry, L. et Soulier, P. (dir.) : Les approches spatiales des méthodes statistiques appliquées à l'archéologie. Journée d'étude organisée par le 13 décembre 2007 à la MAE de Nanterre. Cahiers transversaux de l'UMR 7041

Johnson, A.W. et Earle, T. 1997, 2000 (2e éd). The Evolution of Human Societies. From Foraging Group to Agrarian State. Stanford, Stanford University Press, 456 p.

JoLy O. 1999. Geographical position : State of french art of spatial accessibility indicators. SPESD France, Groupe de travail I. 1, 23 p. 
Journées intenationales d'histoire. Centre culturel de l'abbaye de Flaran, 1982. «L'homme et la route en Europe occidentale au Moyen Age et aux Temps Modernes ", in : Cahier de Flaran, $n^{\circ} 2$, Auch. 303 p.

Jullian, C. 1920. Histoire de la Gaule, tome V, Paris Hachette, 381 p.

JuNG, C. 1999. Morphogénèse, fonctions et évolution de la centuriation B d'Orange et essai de restitution diachronique des paléopaysages du Tricastin (Drôme-Vaucluse). Thèse de doctorat, Université de Tours, $366 \mathrm{p}$.

Konvitz J.F. 1987. Cartography in France, 1660-1848, Science, Engineering and Statecraft, Chicago/ London, University of Chicago press. 194 p.

LAFfont, P. Y et Bréchon, F. 2008. «Châteaux, incastellamento et réseau viaire au Moyen-Age en France méridionale », in : Conpatangelo-Soussignan R., Bertrand J. R., Chapman J. et Laffont P. Y. (dir.). Marqueurs des paysages et systèmes socio-économiques. Actes du colloque COST du Mans (7-9 décembre 2006), Rennes, Presses Universitaires, 2008 : 149-163.

Lамвот, B. 1996. «Les Rèmes à la veille de la romanisation. Le porcien au Ier siècle avant J. C. », in : Bayard D. et Collard J. L. (dir.), Revue Archéologique de Picardie, $n^{\circ}$ spécial, 11/1996, Amiens : 1338.

Lavigne, C. 1997. «Parcellaires de fondation et parcellaires de formation à l'époque médiévale en Gascogne. Clefs de lecture et problèmes d'interprétation », in : CHOUQuer G. (dir.) 1997 Les Formes du paysage, Tome 3 : L'analyse des systèmes spatiaux, Paris, Editions Errance : 149-159.

LebecQ, S. 1989. «La Neustrie et la mer », in : H. Atsma (publ.), La Neustrie, Les pays au nord de la Loire de 650 à 850. Jan Thorbecke Verlag Sigmaringen, 1989 : 406-440.

Legros, R. 1983. «Les chemins des Rèmes », in : Chevallier R. (dir.) 1983 Les voies anciennes en Gaule et dans le monde romain occidental, Colloque tenu à Paris, ENS, 5-6 juin $1982 »$. Caesarodunum $n^{\circ} X V I I I$, Tours, Université de Tours : 269-289.

Leone, F., Aste, J.-P., Velasquez, E. 1995. «Contribution des constats d'endommagement au développement d'une méthodologie d'évaluation de la vulnérabilité appliquée aux phénomènes de mouvements de terrain ». Bulletin de l'Association de Géographes Français - « Croissance urbaine et risques naturels », Vol. 4, 72ème année : 350-371.

LePETIT, B. 1984. Chemins de terre \& voies d'eau, réseaux de transport organisation de l'espace, Éds. de l'E.H.E.S.S., Paris. 148 p.

Lepetit, B. 1988. Les villes dans la France moderne (1740-1840), Paris, Albin Michel. 490 p.

Leturce, S. 1997. «La route et le paysage. Dynamique et stabilité des réseaux routiers beaucerons entre Etampes et la forêt d'Orléans », in : Chouquer G. (dir.) 1997 Les Formes du paysage, Tome 3 : 
L'analyse des systèmes spatiaux, Paris, Editions Errance: 66-78.

LeURent, F. 2006. Structures de réseau et modèles de cheminement. Lavoisier, 394 p.

Levy, J. et Lussault, M. (dir.), 2003. Dictionnaire de la géographie et de l'espace des sociétés. Paris, Belin, 1033 p.

Livet, G. 2003. Histoire des routes et des transports en Europe. Des chemins de Saint-Jacques à l'âge d'or des diligences. Strasbourg, Presses universitaires, 608 p.

LLobera, M. 2003. " Estending GIS-based visual analysis: the concept of visualscapes ». International Journal of Geographical Information Science Vol. 17/1 : 25- 48.

Lot, F. et Fawtier, R. 1932. Le premier budget de la monarchie française, Paris, 259 p.

Loubès, G., « Routes de la Gascogne médiévale », Journées intenationales d’histoire. Centre culturel DE L'ABbaye de Flaran, 1982. «L'homme et la route en Europe occidentale au Moyen Age et aux Temps Modernes », in : Cahier de Flaran, $n^{\circ}$, Auch : 33-56.

Luchaire, A. 1885. Les actes de Louis VII, Paris, Editions Alphonse Picard, 527 p.

Lundmark, H. 1984. « The identification of tribal hierarchies », in : K. Kritiansen (éd.), Settlement and Economy in Later Scandinavian Prehistory, British Archaeological Reports International, Series 211 : 43-62.

Malrain, F., Matterne, V., Méniel, P. 2002. Les paysans gaulois. Paris, Errance, 2002, 236 p.

Marchand, C. 1997. «Réseau viaire et dessin parcellaire : étude morphologique de la région du Gâtinais oriental. » In Chouquer (dir.) 1997, Les Formes du paysage, Tome 3 : L'analyse des systèmes spatiaux, Paris, Editions Errance, 66-77.

Marchand, C. 2000. «Recherches sur les réseaux de formes. Processus dynamiques des paysages du sénonais occidental », thèse de doctorat, Université de Tours. 2 vol.

Marchand, C. 2003. « Des centuriations plus belles que jamais ? Proposition d'un modèle dynamique d'organisation des formes », Études Rurales, juillet décembre 2003, n 167-168 : 93-114.

Mathis, Ph. (dir.) 2003. Graphes et réseaux, modélisation multiniveau. Paris, Lavoisier, 361 p.

Mazoyer, M. et Roudart, L. 1997, 2002 (rééd). Histoire des agricultures du monde : du néolithique à la crise contemporaine, Paris, Seuil, 705 p.

Mennessier-Jouannet, C. et Buchsenschutz, O. 1996. « Des « champs celtiques » aux systèmes de parcellaire protohistoriques », in : ChOuquer G. (dir.) 1996. Les Formes du paysage, Tome I : études sur les parcellaires : pré-actes du colloque d'Orléans (mars 1996). Paris, éditions Errance : 174-180.

Ministère de L'Environnement et du CAdRe de Vie 1980. Lutte contre les inondations - Méthode 
sommaire d'évaluation des critères économiques. Direction de la Prévention des Pollutions - Service de l'Eau - Sous-Direction des Eaux Continentales, 75 p. annexes

Mordant, C., Saligny, L. Poulet-Crovisier, N. «La circulation des objets métalliques à l'âge du Bronze entre Rhône et Saône: utilisation d'une transformation cartographique linéaire », Mappemonde $n^{\circ} 83$ (3-2006), en ligne sur http://mappemonde.mgm.fr.

Muraco, W.A. 1972. «Intraurban accessibility ». Economic Geography, 48 : 388-405.

Nordman, D. 1990. «La connaissance géographique de l'État (XIV $-X V I I^{\mathrm{e}}$ siècles) », in L'État moderne : le droit, l'espace et les forme de l'État, Paris, CNRS, 199 : 175-188.

Nuninger, L. et SAnders, L. 2006. «La modélisation des réseaux d'habitat en archéologie: trois expériences », Mappemonde ${ }^{\circ} 83$ (3-2006), en ligne sur http://mappemonde.mgm.fr.

Ouzoulias, P. 1991. «Eudes Rigaud et le vieux chemin Paris-Rouen », in : Cuisenier J. (dir.), 1991. Matière et figure. Paris, La Documentation française, Collection études et Travaux n³ : 17-42.

Ozouf-Marignier M.-V. et VerdiER N. 2000. "L'événement: un objet historique à emprunter", L'Espace Géographique, ${ }^{\circ} 3,218-223$.

Parish, Y.I.H., Müller, P. 2001. «Procedural Modeling of Cities», in : Proceedings of ACM SIGGRAPH 2001, ACM Press / ACM SIGGRAPH, New York. E. Fiume (ed), Computer Graphics, Annual Conference Series, ACM : 301-308.

Pelletier, M. 2002. Les cartes des Cassini, La science au service de l'État et des régions, Paris, CTHS. 338 p.

Pérol, C. 2004. « Cheminement médiéval, l’homme l'historien et la route », in Fray, J.L. et Pérol, C. L'historien en quête d'espaces, Clermont-Ferrrand, Presses Universitaires Blaise Pascal : 91-107.

Petitfrère C. 2003. « Dessine-moi un village... Le « village » sur la carte et le plan en Limousin sous l'Ancien Régime », in Tricard Jean (dir.), Le Village des Limousins : Études sur l'habitat et la société rurale du Moyen Âge à nos jours, Limoges, Presses universitaires de Limoges. 257-272.

Picon, A. 1992. L'invention de l'ingénieur moderne : l'École des ponts et chaussées, 1747-1851, Paris, Presses de l'École des ponts et chaussées. 767 p.

Picon, A. et Yvon, M. 1989. L'ingénieur artiste, Dessins anciens de l'École des ponts et chaussées, Paris, Presses de l'École des ponts et chaussées. 206 p.

Pinchemel P. 1944. « Habitat Rural et Maisons Rurales en Picardie », Bulletin de l'association des géographes français, $\mathrm{n}^{\circ}$ 163-166. 102-108.

Pinchemel, P. et Pinchemel, G., 1988. La face de la terre, éléments de géographie. Paris, Armand Colin, 515 p. 
Pion, P. 1990. « De la chefferie à l'État ? Territoires et organisation sociale dans la vallée de l'Aisne aux Âges des métaux (2200-20 av. J-C.) », Archéologie et Espaces, Actes des Rencontres Internationales d'Archéologie et d'Histoire d'Antibes (10; 1989), Editions APDCA, Antibes : 183260.

PiтTs, F. 1965. « A graph theoretic approach to historical geography ». The Professional Geographer, $\mathrm{n}^{\circ} 17: 15-20$.

Prou, M. 1884. Les coutumes de Lorris, Paris, 176 p.

Revel, J. 1992. « La région », in, Les lieux de mémoire, III, Les France, 1, Conflits et partages, nrf, Gallimard : 850-883.

Robert, S. (à paraître). «Des outils pour l'analyse de la transmission des trames et réseaux planimétriques : approches multiscalaires et traitements sur SIG», in: direct Colloque du réseau thématique pluridisciplinaire «Paysage et environnement, de la reconstitution du passé aux modèles prospectifs », Chilhac 27-30 sept. 2006.

Robert, S. 1996. «Le Parcellaire du plateau de Sénart (Seine-et-Marne)», in : Chouquer G. (dir.) 1996 : Les Formes du paysage, Tome I : études sur les parcellaires : pré-actes du colloque d'Orléans (mars 1996). Paris, éditions Errance : 11-27.

RoBert, S. 1997. «Le relevé parcellaire sur photographies aériennes et cartes anciennes », in : Chouquer G. (dir.) 1997. Les Formes du paysage, Tome 3 : L'analyse des systèmes spatiaux, Paris, Editions Errance : 88-95

Roвert, S. 2004. L'analyse morphologique des paysages entre archéologie, urbanisme et aménagement du territoire. Exemples d'études de formes urbaines et rurales dans le val d'Oise, thèse de doctorat, Université de Paris 1, 2 vol.

RовеRт, S. 2006a. «La résilience des réseaux routiers : l'exemple du Val-d'Oise », in : Bulletin AGER $n^{\circ} 15$, année 2005, Besançon, Presses Universitaires de Franche-Comté, 2006 : 8-14.

Roвert, S. 2006b. «Les itinéraires anciens traversant le département du Val-d'Oise », in : Bulletin archéologique du Vexin Français et du Val-d'Oise, $n^{\circ} 38$ : 7-23

Robert, S. 2007. « Modeling Ancient Roads », in : Medieval Europe Paris 2007, 4e Congrès international d'Archéologie Médiévale, Institut National d'Histoire de l'Art, Paris, 3-8 septembre 2007. en ligne sur http://medieval-europe-paris-2007.univ-paris1.fr.

Roncayolo, M. 1988. "la morphologie, entre la matière et le social", Villes en parallèle, n¹2-13, 4259 .

Rouche, M. 1982. «L'héritage de la voirie antique dans la Gaule du haut Moyen-Age (Ve-XIe siècle) », in : Flaran, 1982. «L'homme et la route en Europe occidentale au Moyen Age et aux Temps 
Modernes », in : Cahier de Flaran, $n^{\circ} 2$, Auch. : 12-32.

SAHLINs, P. 1996. Frontières et identités nationales, la France et l'Espagne dans les Pyrénées depuis le XVIIe siècle, Paris, Belin ( $1^{\mathrm{er}}$ ed. en anglais 1989), $416 \mathrm{p}$.

Sakarovitch, M. 1984. Optimisation combinatoire. Méthodes mathématiques et algorithmiques. Programmation discrète, Paris, Hermann, 269 p.

SAn Juan, G. 2005. « La Normandie dans l'âge du Bronze européen », in: C. Marcigny, C. Colonna, E. GHESquière et G. VerRon (dir.), La Normandie à l'aube de l'histoire, Les découvertes archéologiques de l'âge du Bronze 2300-800 av. J.-C.. Paris, Somogy : 17.

Schnapp A. 1993. La conquête du passé. Aux origines de l'archéologie. Paris, éditions Carré, 511 p.

SHEFFI, Y. 1985. Urban Transportation Networks - Equilibrium Analysis with Mathematical Programming Methods. Prentice Hall, 400 p.

Spratt, D.A. 1981. "Prehistoric boundaries on the North Yorkshire Moors », in: G. Barker (éd), Prehistoric communities in Northern England : Essays in social and Economic reconstruction, Sheffield, University of Sheffield Press : 87-104.

TISSIER, J.-L. 2003. « Chemins et géographie », Les carnets du paysage, $\mathrm{n}^{\circ} 11: 35-53$

Touzery M. 1995. Atlas de la généralité de Paris au XVIII siècle : un paysage retrouvé, Paris, Comité pour l'histoire économique et financière de la France. 176 p.

Van Leusen, P. M. 2002. Pattern to process : methodological investigations into the formation and interpretation of spatial patterns in archaeological landscapes. Groningen, Rijkuniversiteit. 365 p.

Verdier N. 2002. « Poste et territoires : évolution de la pensée du territoire chez les administrateurs de la Poste au XIXème siècle ", in : Le Roux (dir), Histoire de la poste. De l'administration à l'entreprise, Editions rue d'Ulm : 61-86.

Verdier N. 2004. "L'échelle dans quelques sciences sociales : petite histoire d'une absence d'interdisciplinarité », in O. Orain, D. Pumain, C. Rozemblat et N. Verdier, Géographie, échelles et temporalités en géographie, Paris, CNED : 25-56.

Verdier N. 2007. " Le réseau technique est-il un impensé du XVIIIe siècle : le cas de la poste aux chevaux », Flux, Cahiers scientifiques internationaux Réseaux et Territoires, 68 : 7-21.

VERDIER N. 2008a. « Gerarchie urbane e città in rete : l'urbanizzazione della Francia tra 1700 e 1830 », in Iachello E. e Militello P. (dir.), L'insediamento nella Sicillia d'étà moderna et contemporanea, Bari, Epuglia.

Verdier N. 2008b. "Modeler le territoire : les ingénieurs des Ponts et chaussées et leurs usages de la carte (fin XVIIe-début XIXe siècles)", in Isabelle Laboulais (dir.), Les usages des cartes (XVIIe-XIXe 
siècles, pour une approche pragmatique des productions cartographiques, Strasbourg, Presses universitaires de Strasbourg, 2008, 51-66 et VI-VII.

Verhagen P., Gili S., Mico R., Risch R. 1999. " Modelling Prehistoric Land Use Distribution in the Rio Aguas Valley (SE Spain) », in: Dingwall L. et al. (eds) : Archaeology in the Age of the Internet. Proceedings of the CAA97 conference, Oxford, BAR International Series, 750, CDRom.

Vidal De Lablache, P., 1902. "Routes et chemins de l'ancienne France », Bulletin de géographie historique et descriptive, $\mathrm{n}^{\circ} 17: 115-126$.

Vidal, L., Pomarèdes, H. et Sauvage, L. 1996. «Traces matérielles de la mise en valeur et de l'aménagement des campagnes aux portes de Nîmes : les parcellaires antiques de la plaine du Vistre », in : Chouquer G. (dir.) : Les Formes du paysage, Tome II : études sur les parcellaires : actes du colloque d'Orléans (mars 1996). Paris, éditions Errance, 1996 : 57-66.

VIGNON E. 1862. Études sur l'administration des voies publiques en France aux dix-septième et dixhuitième siècles, Paris, Dunod. 3 vol. XIII-146-145, 213-358, 135-281 p.

Vion, E. 1989. «L'analyse archéologique des réseaux routier : une rupture méthodologique, des réponses nouvelles », Paysages découverts $I:$ 67-69.

Vita-Finzi, C. et Higgs, E.S. 1970. «Prehistoric economy in the Mount Carmel area of Palestine: site catchment analysis », Proceedings of the Prehistoric Society, $36: 1-37$.

Voyages et Voyageurs au Moyen Age, 1996. Société des historiens médiévistes de l'enseignement supérieur public. Voyages et Voyageurs au Moyen Age. XXVIe Congrès de la SHMES (LimogesAubazine, mai 1995), Paris, Publications de la Sorbonne, 314 p.

Wabont, M., Abert, F., Vermeersch, D. 2006. Le Val-d'Oise - 95. In : Provost M. (dir.), Carte Archéologique de la Gaule. Le département du Val-d'Oise. Paris : Editions Les Belles Lettres, 495 p.

Wasserman, S., Faust, K. 1994. Social Network Analysis - Methods and applications. Cambridge University Press, $857 \mathrm{p}$.

Watteaux, M. 2004. «Le plan radio-quadrillé des terroirs non planifiés », in : Chouquer G. (prés.). Etudes Rurales $n^{\circ} 167-168.2004$ : 187-214.

Weber, J. 2000. «Using GIS to Model and Visualize Congestion Effects on Individual Accessibility », in : Proceedings of the Annual Meeting of the East Lakes and West Lakes Divisions of the Association of American Geographers, Oxford, Ohio, 18 p.

Zaksek, K., Fovet, E., Nuninger, L. et Podobnikar T. 2007. " Path modelling and settlement pattern », in : Computer Applications and Quantitative methods in Archaeology, Berlin, , Germany, April 2-6, 2007. Bonn (Germany): Habelt (Kolloquien zur Vor- und Frühgeschichte, vol. 10. 